\title{
A Framework to Service and Network Resource Management in Composite Radio Environments 1
}

\author{
L.-M. Papadopoulou, V. Stavroulaki, P. Demestichas, and M. Theologou \\ National Technical University of Athens (NTUA), \\ Electrical and Computer Engineering Department, Telecommunications Laboratory, \\ 9 Heroon Polytechneiou Str, 15773 Zographou, Athens, Greece \\ louisa@telecom.ntua.gr
}

\begin{abstract}
This paper builds on the assumption that in the future, UMTS, HIPERLAN-2 and DVB-T can be three (co-operating) components of a composite radio infrastructure that offers wideband wireless access to broadband IP-based services. Managing the resources of this powerful, composite-radio infrastructure in an aggregate manner, and multi-operator scenario, is a complex task. This paper presents an approach to the overall UMTS, HIPERLAN-2 and DVB-T network and service management problem, providing the internal operation of a system addressing this problem. Key points addressed are the development of an architecture that can jointly optimise the resources of the technologies in the composite radio environment, and the development of open interfaces with Service Provider mechanisms and the heterogeneous managed infrastructure.
\end{abstract}

\section{Introduction}

Wireless systems continue to attract immense research and development effort [1], especially in the following areas. First, the gradual introduction of third generation systems like the Universal Mobile Telecommunications System (UMTS) [2] and the development of the IMT-2000 framework [3]. Second, the standardisation, development and introduction of Fixed Wireless Access (FWA) systems, which support radio access to broadband services, with limited mobility; a pertinent promising example is the HIPERLAN (High Performance LAN) initiative [1]. Third, the advent of Digital Broadcasting Systems (DBS) like the Digital Video Broadcasting (DVB) and the Digital Audio Broadcasting (DAB) initiatives [4]. Moreover, a recent trend (compliant with the features envisaged for the Fourth Generation (4G) wireless systems' era) is to assume that UMTS, HIPERLAN-2 and DVB-T will be three co-operating wireless access components.

In other words, UMTS, HIPERLAN-2, and DVB-T can be seen as parts of a powerful, composite-radio, infrastructure through which their operators will be

1 Work partially funded by the CEC, under the $5^{\text {th }}$ Framework Program, within the IST project MONASIDRE (IST-2000-26144: Management of Networks and Services in a Diversified Radio Environment). 
enabled to provide users and service providers (SPs) with alternatives regarding the efficient (in terms of cost and QoS) wireless access to broadband IP-based services. This paper presents the development of a UMTS, HIPERLAN-2 and DVB-T network and service management system capable of:

- Monitoring and analysing the statistical performance and QoS levels provided by the network elements (segments) of the managed infrastructure, and the associated requirements originating from the service area (environment conditions, e.g., traffic load, mobility levels, etc.).

- Inter-working with SP mechanisms, so as to allow SPs to dynamically request the reservation (release, etc.) of network resources.

- Performing dynamic reconfigurations of the overall managed UMTS, HIPERLAN2 and DVB-T infrastructure, as a result of resource management strategies, for handling new environment conditions and SP requests in a cost-efficient manner.

In the following, the management architecture in the aspect of a composite radio and multi-operator context, and the operation of such a system are presented.

\section{Management Architecture in a Composite Radio and Multi-operator Context}

Our model of the composite radio environment includes three different wireless access technologies and has a flexible implementation. In the context of this paper, each wireless access system is considered to belong to a different operator, occupying a network and service management platform. A generic management architecture of such a platform is split in three logical entities as follows.

- MASPI (Monitoring and Assessment and SP mechanism Interworking). This component captures the (changing with time) conditions that originate from the environment (service area) of the managed UMTS, HIPERLAN-2 and DVB-T infrastructure; this is accomplished by monitoring and assessing the relevant network and service level performance of the managed network elements and segments. This component also interworks with the SP mechanisms, so as to allow SPs to request the reservation of resources (establishment of virtual networks) over the managed network infrastructure. Virtual networks are seen as the realisation of contracts that the managed system should maintain with SPs.

- RMS (Resource Management Strategies). This component applies resource management strategies, so as to dynamically find and impose the appropriate UMTS, HIPERLAN-2 and DVB-T infrastructure reconfigurations, through which the service provider requests, and/or the (new) service area conditions, will be handled in the most cost-efficient manner.

- NES (UMTS, HIPERLAN-2 and DVB-T Network and Environment Simulator). It provides the means for validating some management decisions prior to their application in the real network. This component enables off line testing, validation and demonstration of the management mechanisms. 
The management system components are distributed in each domain, specialised for handling the specific (UMTS, HIPERLAN-2 and DVB-T) technology. However, these components can co-operate for handling SP requests and/or new environment conditions.

\section{System Operation}

A sample scenario according to which the components above collaborate is provided in Fig. 1. MASPI-U, RMS-U, and NES-U represent components dedicated to the UMTS network (similarly for the HIPERLAN-2 and DVB-T networks). The interactions with the NES-U, NES-H and NES-D components are omitted for simplicity reasons.

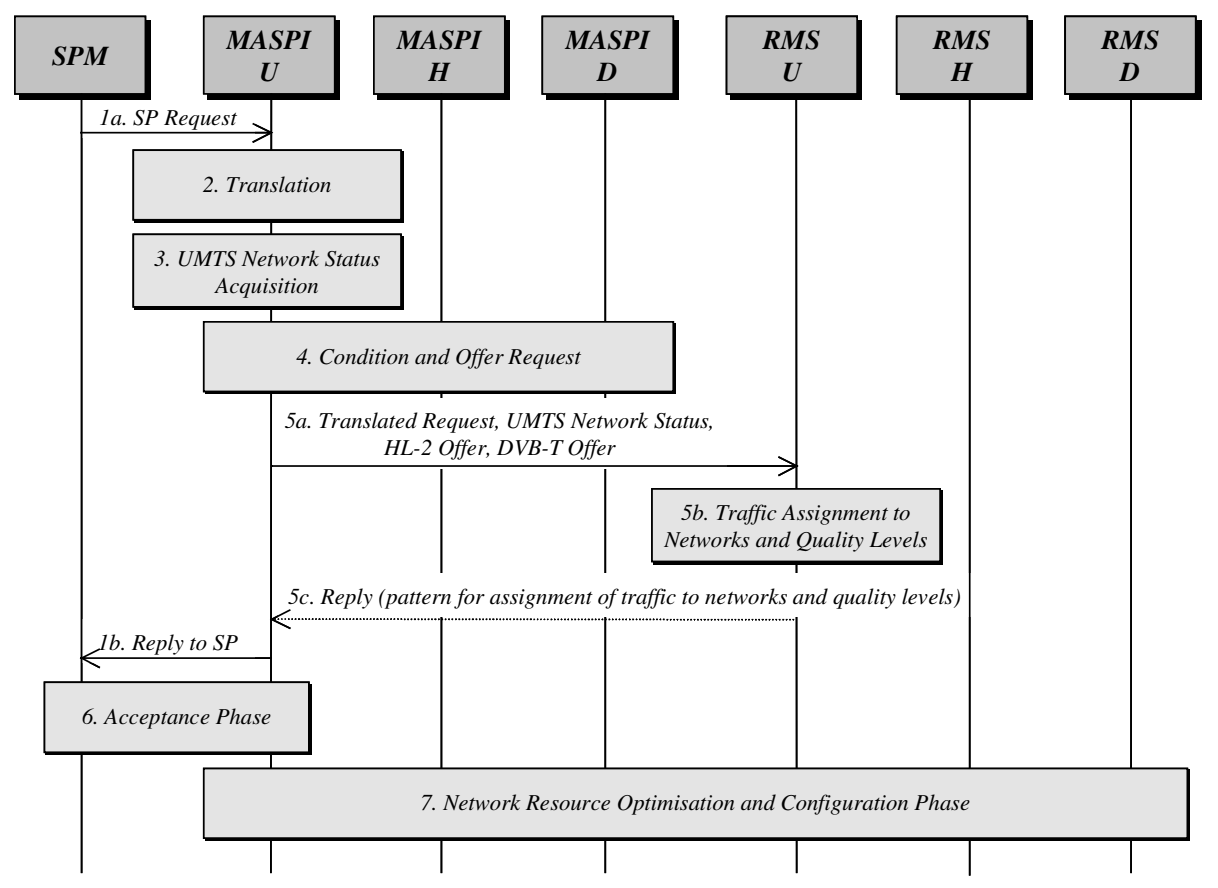

Fig. 1. Sample operation scenario. The scenario shows how the components of the UMTS, HIPERLAN-2 and DVB-T network and service management system collaborate

The initiation of the scenario is done from the UMTS network, as an example. The procedure would be similar if the MASPI component observed a new environment condition (e.g., alteration in the traffic demand, mobility and interference levels, etc.) in the managed network. Alternatively, the process could have been initiated by a SP request towards the HIPERLAN-2 or DVB-T management system. The network that 
receives a SP request or observes a new environment condition is called originating network.

The scenario consists of steps that can be roughly categorised in four phases. In the first phase (step 1), the SP issues a virtual network establishment request. In the second phase (steps 2-5), the request is processed by the UMTS, HIPERLAN-2 and DVB-T network and service management system (this includes translation of the SP request from a service to a network level view, network status acquisition of the originating network, condition and offers from the co-operating networks, and traffic assignment to networks and quality levels). In the third phase the proposed solution is accepted by the parties involved (SP and network operators, step 6). Finally, in the fourth phase the managed systems are appropriately configured (step 7). The aforementioned steps are addressed in detail in the following sub-sections.

\subsection{Service Provider Request}

MASPI supports the functionality of handling the SP requests and the corresponding replies to these requests, as a general framework of the processing, establishment and maintenance of contracts (SLAs) with the SPs. A typical SP request has the following general structure (content). (i) It specifies the service (or set of services), the provision of which the SP requests from the management system. (ii) It specifies the distinguishable user classes to which the service is offered; each user class is associated with specific quality levels, a user class profile and a terminal profile. The quality of service levels express the quality levels that are considered acceptable for the provision of services to users (subscribers) that belong to the specific user class. If the quality levels are more than one, the SP may also provide the significance factor of each quality level for the present user class. The user class profile includes mobility, and traffic characteristics of the users, and is described in a file with specific format. The terminal profile assists the management system on knowing which networks can be used to satisfy the SP request (e.g., users of a specific user class may have terminals that support only the UMTS network). (iii) It specifies the number of subscribers that correspond to each service and user class. (iv) It includes information about the area (geographic region) to which the request is applied to, and the time zone, i.e., the time period during the day that the service should be provided to the users of the specific user class.

\subsection{Service Provider Request Translation}

MASPI supports the functionality of translating the SP request from a service level view to a network level view. The information about the requested services, and user classes (including user class profiles and quality of service levels) is used in order to investigate various options regarding the network load required to fulfill the request. On the other hand, the area information in the SP request can be exploited for the detection of the cells that will be affected by the SP request. 


\subsection{Network Status Acquisition}

During this task the status of the originating network (e.g., traffic carried by cells that can be affected by the SP request) is obtained. MASPI maintains interfaces with the underlying network interface and/or the network element management infrastructure. MASPI collects service level information based on the handled load, the provided performance (measured e.g., by the blocking probability, the dropping probability or the delay), and the dedicated resources per service provider, service, and user class. An integration of this service level information about the management system enables a network level provisioning of the managed system infrastructure. In case of performance degradations MASPI can initiate the procedure of the scenario described in Fig. 1.

\subsection{Condition and Offer Request}

Each MASPI is capable of requesting from the co-operating networks' MASPIs the amount of resources or the load that these networks can carry, as well as cost related information. Likewise, each MASPI is in position to respond to such requests. This information (bandwidth availability and cost), as well as other information (e.g., the area and time zone for which the services should be provided), will contribute to the decision of the traffic splitting between the three networks.

\subsection{Traffic Assignment to Networks and Quality Levels}

This is an optimisation problem, targeted to the splitting of the traffic to the three networks and the assignment to quality levels. Considering the case of the scenario depicted in Fig. 1, this optimisation problem relies on the following input data:

- The translated SP request, which can express the service demand per user class;

- The benefit deriving from the assignment of (portions of the) service demand to the several quality levels;

- The status of the UMTS, HIPERLAN-2 and DVB-T network segments that are to be affected by the request;

- The UMTS, HIPERLAN-2 and DVB-T offers, i.e., the cost that these networks will impose per quality level of the service.

The optimisation results to an allocation of the service demand to networks and quality levels. The allocation should optimise an objective function, which is associated with the amount of the service demand accommodated, the quality levels at which the service demand will be accommodated, and the benefit deriving from the assignment of service demand to high quality levels.

The constraints of the optimisation problem fall into the following categories:

- The service demand should be assigned to acceptable quality levels;

- The capacity constraints (deriving form the UMTS network status, and the HIPERLAN-2 and DVB-T condition and offers) should not be violated. 


\subsection{Reply to Service Provider Request - Acceptance Phase}

The MASPI component reply to the initial SP request includes the quality levels to which the user classes are assigned, cost related information per user class, as well as the volume of the subscribers assigned to each network. This information is valid for the area and time zone specified in the SP request. The acceptance of this reply from the SP will lead to the establishment of a SLA.

\subsection{Network Resource Optimisation and Configuration}

After the decision by the RMS on the traffic allocation to the three networks, and the acceptance phase, the MASPI of the originating network makes a resource reservation request to the other networks' MASPIs, in order these systems to accommodate the assigned traffic. Thereafter, optimisation and configuration procedures take place among the three networks. RMS finds an optimal configuration of the managed network segments, so as to guarantee that the traffic assigned to them will be handled (carried) with the most cost-efficient manner. This part of the management system consists in a suite of tools and procedures that optimise functions including for instance cost, network performance criteria, etc., under a set of constraints related to target QoS levels, resource utilisation, fault tolerance, etc.

\section{Conclusions}

This paper builds on the assumption that in the future, UMTS, HIPERLAN-2 and DVB-T can be three (co-operating) components of a composite radio infrastructure that offers wideband wireless access to broadband IP-based services. In this direction the paper presented an approach to the overall UMTS, HIPERLAN-2 and DVB-T network and service management problem, addressing the operation of a system that deals with such a problem.

The paper, or alternate versions of this paper, can be expanded with more information on the internal functionality of the components, the provision of details regarding the design choices followed, or the presentation of indicative results obtained from case studies. The application of the management architecture in largescale network test-beds is a future stage of our work.

\section{References}

1. U. Varshney, R. Vetter, "Emerging mobile and broadband wireless networks", Commun. of the ACM, Vol. 43, No. 6, June 2000

2. "Wideband CDMA", Feature topic in IEEE Commun. Mag., Vol. 36, No. 9, Sept. 1998

3. "IMT-2000: Standards effort of the ITU", Special issue on IEEE Personal Commun., Vol. 4, No. 4, Aug. 1997

4. Digital Video Broadcasting Web site, www.dvb.org Jan. 2001 